\title{
ENLACES, CRES y Regionalidad: algunas aproximaciones sobre la integración de los campos de Educación Superior en América Latina y el Caribe
}

\author{
ENLACES, CRES and Regionness: some approaches on the integration of Higher Education fields in Latin \\ America and the Caribbean
}

Mário Luiz Neves de Azevedo

Professor na UEM e pesquisador do CNPq (Brasil), Brasil

mlnazevedo@uem.br

\section{Resumen:}

Este artículo analiza la formación de ENLACES (Espacio de Encuentro Latinoamericano y Caribeño de Educación Superior), previsto en la Declaración de la Conferencia Regional sobre Educación Superior en América Latina y el Caribe - CRES 2008. El artículo también trata sobre regionalismo, regionalización y regionalidad. Para esto, metodológicamente, se utilizan teorías críticas de enfoque, como la Teoría de los Campos Sociales de Pierre Bourdieu, el Nuevo Regionalismo de Björn Hettne, Frederik Söderbaum y Tanja A. Börzel, y la Economía Política Cultural (CPE), especialmente de Roger Dale y Susan Robertson.

Palabras ClaVe: ENLACES, Educación Superior, Regionalización, Regionalidad, CRES.

\section{Abstract:}

This paper analyzes the formation of ENLACES, foreseen in the Declaration of the Regional Conference on Higher Education in Latin America and the Caribbean - CRES 2008. The article also deals with regionalism, regionalization and regionness. For this, methodologically, critical theories of approach are used, such as Pierre Bourdieu's Theory of Social Fields, the New Regionalism of Björn Hettne, Frederik Söderbaum and Tanja A. Börzel, and Cultural Political Economy (CPE), especially from Roger Dale and Susan Robertson.

KEYWORDS: ENLACES, Higher Education, Regionalization, Regionness, CRES.

\section{INTRODUCCIÓN}

La Conferencia Regional de Educación Superior en América Latina y el Caribe (CRES) 2018, se realizó diez años después de la CRES 2008 y 22 años después de la CRES 1996, que fuera el primer encuentro regional de actores sociales relacionados con la Educación Superior). Las tres ediciones de la CRES fueron promovidas por el Instituto Internacional de la UNESCO para la Educación Superior en América Latina y el Caribe,(IESALC-UNESCO), con diversos apoyos, incluso de universidades y ministerios de educación de ciencia y tecnología de países de la región.

A pesar de las buenas intenciones expresadas en la Declaración de la CRES 2018 realizada en la histórica Universidad Nacional de Córdoba (UNC, Argentina), en ocasión del centésimo aniversario del fecundo Movimiento de Córdoba de 1918, no puede decirse preliminarmente que hubo avances significativos en relación con lo que fuera declarado en Cartagena de Indias (Colombia) en el marco la CRES 2008. Especialmente, en lo que se refiere al tema de la integración regional y la consecución del Espacio de Encuentro Latinoamericano y Caribeño de Educación Superior (ENLACES), que era un programa necesario para la integración regional y para la internacionalización de la Educación Superior.

De acuerdo con lo consignado por los participantes de la CRES 2008 en la Declaración pública realizada en la clausura del encuentro, la construcción del ENLACES sería fundamental para lograr la integración de la región. Para ello, el ENLACES debería "formar parte de la agenda de los gobiernos y de los organismos multilaterales de carácter regional" (Declaración CRES, 2008, p. 11). 
Sin dudas, algo tan crucial para la integración regional suscita la cuestión respecto de "¿qué es el ENLACES?". Para esbozar alguna respuesta, es posible recurrir inicialmente al portal de ENLACES, ubicado en el sitio de internet del IESALC-UNESCO, dedicado desde 2008 a esta política regional de integración académica de América Latina y el Caribe:

El Espacio de Encuentro Latinoamericano y Caribeño de Educación Superior (ENLACES) es una plataforma regional para la movilización y articulación de acciones concretas de cooperación académica solidaria que promuevan el conocimiento de las características, tendencias y problemas de la Educación Superior en la región; el diálogo entre los actores institucionales con miras a la consolidación de una agenda de consenso encaminadas a la superación de nuestras deficiencias y la promoción de una Educación Superior inclusiva, de calidad y pertinente; el apoyo a los procesos de reforma y/o fortalecimiento de los sistemas nacionales y de las instituciones de Educación Superior y la divulgación de informaciones vinculados a esos procesos (IESALC-UNESCO, 2008).

Para dar curso a lo acordado en 2008, el IESALC de la UNESCO, fiel depositario y principal promotor de lo acordado en la CRES 2008, creó una "Comisión de Seguimiento" mandato suficiente para encaminar diligencias, acompañar cronogramas, verificar la ejecución efectiva y tomar las debidas providencias en nombre del IESALC y de los delegados de la CRES 2008. La intención era exigir el cumplimiento de aquello que fuera decidido y registrado en la Declaración y en el Plan de Acción de la CRES 2008. Así lo percibió Marco Antonio Días, para quien hubiera sido necesario el nombramiento de un comité de aplicación, para acompañar el cumplimiento de las recomendaciones de la Conferencia General de la UNESCO de París (,1993) referida a convenios regionales para la convalidación de títulos y diplomas. En sus palabras, "lo que faltó en la Recomendación [en 1993] fue prever la existencia de un comité encargado de su aplicación. Este comité debe tener representantes de todas las regiones y, naturalmente, de las universidades a través de sus asociaciones"(Días, 2016, p. 66).

La formación de un Comité de Aplicación o de un grupo de trabajo para el cumplimiento de la CRES 2008 y de su respectivo Plan de Acción sería una innovación política para el seguimiento y la efectividad de lo contenido en la II Declaración y en los consecuentes acuerdos establecidos entre la UNESCO, los Estados Nacionales y demás actores sociales en favor del avance de la Integración regional latinoamericana y caribeña. Algo que puede realizarse, en esta oportunidad, a partir de la CRES 2018 - es decir, si el correspondiente Plan de Acción contemplara el ENLACES como estrategia de integración.

En este sentido, al examinar la Declaración de la III CRES 2018, se vuelve evidente que no hay mención al ENLACES ni tampoco se mencionan los gobiernos nacionales, las redes y organizaciones según lo acordado en la CRES 2008, que sería esencial para la intención, hecha pública en Córdoba en 2018, de efectividad de la "integración regional solidaria latinoamericana y caribeña" (Declaración CRES, 2018, p. 1) ${ }^{1}$.

Sin embargo, para ilustrar la magnitud de la frustración en la promoción de la integración regional, vale notar lo que destacaron del Valle y Perrotta (2018) en un texto publicado en vísperas de la CRES 2018.Los autores enfatizan los puntos de la Declaración de la CRES 2008, entre ellos el ENLACES:

(...) la imposibilidad de acordar un mecanismo de reconocimiento de titulaciones y trayectos formativos que tenga en cuenta la peculiaridad y la heterogeneidad de los sistemas de Educación Superior de Latinoamérica y el Caribe, las exiguas movilidades académicas intra-regionales - siempre en comparación con la región más gravitante en movilidad regional, la del Espacio Europeo de Educación Superior, sin tomar en cuenta los disímiles puntos de partida regulatorios y de financiamiento disponibles- y la incapacidad de desarrollar y cumplimentar un programa de trabajo para lograr el ENLACES. Al mismo tiempo, respecto de los acuerdos de integración regional y regionalismos, se señala su falta de eficacia para desarrollar políticas de internacionalización (...) (2018, p. 52).

En este sentido, suponiendo que los campos nacionales de Educación Superior signatarios de la Declaración de la CRES 2008 forman parte de un proyecto (casi un proceso) de regionalización en América Latina y el Caribe y que, potencialmente, pueden constituir un campo regional de Educación Superior análogo geográficamente al área de alcance del IESALC, el presente artículo busca debatir sobre el ENLACES y también presenta reflexiones sobre la integración regional europea. 
Para ello, se dedica la próxima sesión a tratar la cuestión del regionalismo, la regionalización y la regionalidad, sobre todo en lo que se refiere a la integración europea. La intención es que pueda comprenderse mejor, por intermedio de la tácita comparación, cómo marcha el proceso de regionalización en América Latina y el Caribe. ${ }^{2}$ Este artículo se apoya metodológicamente en las teorías críticas como la Teoría de los Campos Sociales de Pierre Bourdieu, el Nuevo Regionalismo de Björn Hettne, Frederik Söderbaum y Tanja A. Börzel, y la Economía Política (Cultural Political Economy-CPE), especialmente a partir de Roger Dale y Susan Robertson.

\section{INTEGRACIÓN REGIONAL Y "REGIONALIDAD" 3}

Como muestran Hettne (2005) y Söderbaum (2008; 2015), los proyectos formales de integración regional y los movimientos de regionalización, aparecen de manera más sobresaliente en Europa Occidental tras la Segunda Guerra Mundial, ganando cuerpo en el contexto de la llamada Guerra Fría y la globalización.

No obstante, hay que señalar que, en el resto del mundo, de manera similar, pero con sus singularidades y especificidades, también se ha desarrollado la integración regional. Un ejemplo de ello son la Liga Árabe, la Asociación de Naciones del Sudeste Asiático (ANSEA / ASEAN) y el Mercado Común del Sur (MERCOSUR) (Börzel, 2016). De acuerdo con Robertson, Azevedo y Dale, "la expansión de las actividades inter-estatales a nivel regional y las recientes y nuevas formas de cooperación y organización regional desde la década de 1990 resultaron en la proliferación de conceptos y enfoques"(2016, p. 7). ${ }^{4}$

No es el objetivo de este artículo, abrir un debate sobre las diferentes disciplinas y áreas del conocimiento que se dedican a la cuestión del regionalismo (Ciencia Política, Relaciones Internacionales, Economía, Geografía, Historia, Antropología o Sociología). Tampoco generar una discusión sobre los diferentes enfoques teóricos que buscan la comprensión del regionalismo en las relaciones internacionales, como el funcionalismo, neo-funcionalismo, positivismo, constructivismo, realismo, neorrealismo, liberalismo, neoliberalismo, marxismo o neo-marxismo. La intención es esbozar las discusiones sobre región, regionalismo, regionalización y regionalidad. De esta forma, descartando cualquier sentido eurocéntrico, pero con el fin de establecer una discusión con base histórica, vale la pena hacer algunas aproximaciones sobre la experiencia europea de regionalización.

De esta forma, como percibe Söderbaum (2015), el neo-funcionalismo (variación institucionalista) fue inicialmente la teoría dominante en el movimiento de regionalización en el viejo continente, siendo Ernest Haas el pensador más prominente de esa corriente de las Relaciones Internacionales. Para Söderbaum "los neofuncionalistas resaltaron la imprescindibilidad de instituciones regionales, con miras a ser los medios más eficaces para la solución de problemas comunes" (2015, p. 11).

En la crítica al llamado viejo regionalismo, particularmente el de corte neofuncionalista, Söderbaum (2008), constata que los primeros análisis basados en el nuevo regionalismo fueron desarrollados por Björn Hettne, quien recibe la influencia de Polanyi. Hettne desarrolló un proyecto de investigación en la década de 1980 sobre "la integración europea más allá de la Guerra Fría" (Söderbaum, 2008, p. 2), sin embargo:

(...) el Nuevo Regionalismo fue mencionado por primera vez a mediados de los años 1990 durante otro de los proyectos de
investigación de Hettne: el proyecto UNUWIDER de investigación sobre el Nuevo Regionalismo. El Nuevo Regionalismo
emergió como un esfuerzo ecléctico y exploratorio para comprender el fenómeno regional en el contexto de la globalización
económica. Durante la década de 1990, su foco principal fue sobre los factores sistémicos (especialmente la globalización y el
fin de la Guerra Fría) más que las interdependencias y los factores intrarregionales que caracterizaron muchos de los llamados
enfoques antiguos, especialmente el abordaje neofuncionalista de Ernst Haas y otros (Söderbaum, 2008, p. 3).

La acción de integración a partir del proyecto regional, presenta variadas escalas y dimensiones y puede ser interpretada como un proceso de convergencia política, social, económica y cultural entre diferentes actores sociales, en particular los Estados-naciones, en determinado espacio geográfico, con vistas (como 
estrategia común de los Estados miembros y otros actores) a la coordinación de políticas. Pero también, la regionalización propicia la formación de un campo social regional, involucrando intereses de orden económico, político y cultural que, ampliando aún más el abordaje del Nuevo Regionalismo, puede ser llamado: de campo de poder regional (Bourdieu, 2011).

Una efectiva regionalización, sea la europea o de cualquier otra región, como la latinoamericana y caribeña, requiere como fundamento para su consecución la adecuada y legítima construcción de una identidad regional, denominada por Hettne $(2005)$ y Söderbaum $(2008 ; 2015)$ como regionness (en adelante, "regionalidad" - sin comillas). La regionalidad sería la composición orgánica entre los diversos actores del campo social regional de un espíritu de pertenencia a la región y a los campos sociales en proceso de integración regional -en el caso de este artículo, al campo de la Educación Superior regional que comprendería América Latina y el Caribe.

Este espíritu de pertenencia regional, algo como una ciudadanía orgánica regional, presupone, como enseñaba Karl Polanyi (1980), la incorporación de una cultura integradora (aunque aún en potencia). La existencia y el ejercicio de la regionalidad es parte esencial del fondo de capital cultural en la región (como por ejemplo de lenguas, conocimientos, culturas, artes y ciencias), que los actores sociales, individuales o colectivos, luchan por conquistar (y compartir). Este patrimonio regional común, en sus diversas formas y modalidades de capital cultural, está compuesto por un conjunto de bienes, que en gran medida son bienes públicos distribuidos por los propios estados nacionales en proceso de integración por bienes comunes como la educación, el conocimiento, la ciencia, la cultura, el arte, entre otros, cuyas posesiones denotan pertenencia a el/los campo(s) regional (s) (ya integrados o en proceso de formación). ${ }^{5}$

De esta forma, la regionalidad es un concepto fundamental en el nuevo regionalismo propuesto por Hettne, pues el nivel de regionalidad es lo que demuestra el grado de cohesión social regional (Hettne, 2005) y de la intensidad de integración de los propios actores sociales en sus correspondientes campos sociales regionales. La regionalidad, según Hettne, puede ser tratada como un extenso proceso histórico que, como tal, cambia a lo largo del tiempo, o sea, iniciando por la pura coerción, pasando por la construcción de imperios y naciones y llegando a la cooperación voluntaria y solidaria (Hettne, 2005).

En este sentido la Educación Superior es fundamental en el proceso de regionalización y en la construcción de la regionalidad, en tanto, porción especial del capital cultural que desempeña el papel de cemento ideológico para la cohesión y la integración a escala regional.

Por eso, las Instituciones de Educación Superior y las consecuentes actividades desarrolladas en el campo académico (enseñanza, investigación, tecnología, extensión e innovación), son especialmente convocadas, si no impulsadas, por las autoridades europeas a regionalizarse. Este fenómeno de regionalización académica viene produciéndose, por ejemplo, en Bolonia con el propósito de construir un Espacio Europeo de Educación Superior (EEES) (Dale, 2004; Dale, Robertson, 2009; Azevedo, 2014a y 2014b).

Recurriendo metodológicamente al concepto de campo social de Pierre Bourdieu, la integración regional significará la reconfiguración a escala ampliada -regional- de los campos sociales. De este modo, los actores sociales de los campos específicos nacionales pasan a actuar y a interactuar en espacios regionalmente integrados, compitiendo, luchando, haciendo alianzas, cooperando, disputando objetos y obedeciendo a reglas comunes de conducta. Esto es así, según Bourdieu, porque "un campo es un campo de fuerzas, y un campo de luchas para transformar las relaciones de fuerzas (...)" (Bourdieu, 2011, p. 201).

Esta rutina de acciones es común a los campos sociales, independientemente de las escalas (nacional, regional o global). En otras palabras, incluso integrados a escala regional, los campos sociales continúan siendo campos de fuerzas en que los actores sociales, aunque integrados, luchan para conservar o transformar las relaciones sociales existentes. ${ }^{6}$

La integración regional europea contemporánea, como se mencionó anteriormente, viene siendo referenciada en el neo-funcionalismo de Ernest Haas (1958) ${ }^{7}$ que, por lo demás, parece permanecer, con alguna mitigación de otras escuelas y corrientes de pensamiento, como presupuesto teórico de las autoridades 
europeas para la regionalización en su continente (Robertson et al., 2016). Ernst Haas, con base en el neo-funcionalismo, creía en la idea de que la vida comunitaria regional podría ser promovida por agentes de las élites como, por ejemplo, (policy makers), legisladores, dirigentes políticos y cuadros diversos, que, convencidos de la importancia de la integración regional, aceptarían, legislarían y propagandizarían, al estilo de spill-over, ${ }^{8}$ políticas de integración y el mismo regionalismo (Haas, 1958; Börzel, 2016).

Por lo tanto el regionalismo europeo se ha asentado, históricamente, en tratados y acuerdos entre Estados y ha avanzado como un proceso de aprendizaje para la convivencia e integración de diferentes pueblos. De acuerdo con Laursen (2008), las teorías de la integración se han desarrollado en las relaciones internacionales principalmente para explicar la integración europea. Laursen recuerda también que la integración europea comenzó a principios de los años cincuenta con la Comunidad Europea del Carbón y del Acero (CECA), y subraya que Ernest Haas, en un libro titulado The Uniting of Europe (1958), analizó esta experiencia de integración, siendo el desarrollo del concepto de spill-over su principal contribución teórica [de Haas] (Laursen, 2008, p.3).

Sin la intención de apelar al "nacionalismo metodológico" o al "estatismo metodológico" (Robertson y Dale, 2017), no es ocioso recordar que el proceso de integración regional europea se inició mediante tratados y acuerdos formalizados entre Estados, que cedieron parte de sus soberanías a las autoridades regionales e impulsaron este mismo proceso de regionalización. Por otra parte, esos Estados, fueron los actores sociales, inicialmente, más prominentes en el proceso de regionalización, permitiendo la transferencia de poder y el desplazamiento de la lealtad de sus ciudadanos a autoridades regionales. Para Best y Christiansen la integración regional se realiza por medio de "procesos por los cuales los estados van más allá de la remoción de obstáculos a la interacción entre sus países y crean un espacio regional sujeto a distintas reglas comunes" (2008, p. 436).

Entre las experiencias contemporáneas, la Unión Europea ha sido el bloque regional más sólidamente integrado. 9 Cabe recordar que la integración europea se inaugura formalmente con el Tratado de París, de 18 de abril de 1951, que instituyó la Comunidad Europea del Carbón y del Acero (Tratado CECA), poco después del final de la II Guerra. ${ }^{10}$ Esta primera organización comunitaria es en esencia un acuerdo económico entre Estados para la regulación del sector del acero y el carbón, como principales insumos de la industria de guerra. La hipótesis es que, al regular esta industria de base, se evitaban las hostilidades entre los dos principales contendientes contemporáneos europeos, Alemania y Francia, en tres cuartos de siglo de historia (1870-1945) - Guerra Franco-Prusia o Guerra Franco-Germánica 1870-1871), I Guerra Mundial (1914-1918) y II Guerra Mundial (1939-1945). Esto sin contar las guerras napoleónicas de 1803 a 1815.

La regulación de la producción y distribución del acero y el carbón, como un mercado regional integrado, sería funcionalmente un desestímulo a los conflictos por participación de mercado (market share) y a las guerras como máximas expresiones de la disputa en el campo del poder. ${ }^{11}$ Vale la pena señalar que, en 1950, Jean Monnet (alto cuadro del gobierno francés,- Commissaire Général au Plan), envía un memorandum al Ministro de Asuntos Exteriores de Francia, Robert Schuman, en el que proponía la asociación de las industrias del carbón y el acero y el establecimiento de una "Alta Autoridad" (p. 8) de regulación a ser respetada por Francia y Alemania y por otros países que se unan a esa asociación. La concreción de esta propuesta sería "el primer paso para la fundación de la Federación Europea, algo indispensable para el mantenimiento de la paz" (Comision Europea, 2002, p. 21).

Esquemáticamente, el proceso de integración europea puede dividirse en tres fases. La primera, de 1945 a 1975, se extiende desde la post-Segunda Guerra Mundial, hasta la efectiva percepción de la crisis del capitalismo alrededor de 1975 (período denominado “Años Dorados del capitalismo europeo"), con la intención de impedir la aparición de nuevos conflictos armados. La segunda fase, de 1975 a 2000, se inicia con la crisis del capitalismo y está marcada por las reformas neoliberales, el Nuevo Gerencialismo Público, el avance de la globalización económica, la debacle del socialismo real, el supuesto fin de la Guerra Fría y el lanzamiento de la nueva estrategia de desarrollo europeo en la reunión del Consejo Europeo de Lisboa del 
año 2000. La tercera etapa de la integración europea se inaugura cuando el Consejo Europeo plantea a la Unión Europea el reto de convertirse en la economía de conocimiento más competitiva del mundo. Esto sucede con el lanzamiento del proceso de Lisboa, también conocida como la agenda de Lisboa o la Estrategia de Lisboa (Consejo Europeo de Lisboa, 2000), cuyo sentido sería enfrentar la crisis y avanzar en la integración regional. Para ello, estimuló cambios en el proceso de toma de decisiones y estableció que la promoción de políticas regionales en Europa, en especial para la cuestión del empleo y de la educación, pasaría a ser operada vía Método Abierto de Coordinación (MAC) (Azevedo, 2014a). ${ }^{12}$

La Estrategia de Lisboa, centrada en la mejora de la competitividad europea y en el estímulo a la innovación y la tecnología, parece mantener el neo-funcionalismo como base de las relaciones internacionales de la Unión Europea y se fundamenta ideológicamente en la teoría del capital humano y el modelo de desarrollo propuesto por Joseph Schumpeter (Robertson, et. al., 2016; Schumpeter, 1961 y 1997). ${ }^{13}$

En el marco de esta tercera fase de regionalización europea, se lanza en 2010 la Estrategia Europa 2020, que en esta versión incluye el subtítulo "Estrategia para un crecimiento inteligente, sostenible e inclusivo". El texto fue presentado por la Comisión Europea en el transcurso de la gran recesión desencadenada a partir de diciembre en 2007 con la crisis de los subprimes (crédito a tomadores con garantías menos seguras) y la quiebra del banco de inversión norteamericano Lehman Brothers. ${ }^{14}$

Por lo tanto, como se anunció en la introducción de este artículo, el enfoque del Nuevo Regionalismo en los estudios de las Relaciones Internacionales y las contribuciones de la Economía Política Cultural (Cultural Political Economy - CPE) demuestran que el viejo regionalismo, aquí interpretado en su abordaje neo-funcionalista y asumido por los formuladores de políticas y altos dirigentes europeos, no es suficiente para comprender el fenómeno de la regionalización. Esto ocurre porque, según resaltan Robertson et al. (2016), en tiempos de globalización, el enfoque del Nuevo Regionalismo es fundamental en virtud de las innovaciones conceptuales efectivas y porque este mismo enfoque destaca la región como centro del orden político mundial. ${ }^{15}$

En efecto, la integración regional, que viene siendo fundada en tratados, protocolos y declaraciones de paz, de cooperación, de ayuda mutua y de alianzas, puede ser interpretada, según el abordaje del Nuevo Regionalismo y con base en la Economía Política Cultural (Cultural Political Economy - CPE) (Robertson, et. al, 2016) y en la teoría de los campos de Bourdieu, como un conjunto de procesos que involucran embates, combates, debates y consensos que vienen siendo formalizados en variados diplomas intergubernamentales y comunitarios (después, internalizados en la legislación de cada Estado Parte), institucionalizados por la fundación de autoridades regionales, consolidados por la movilidad de capital y trabajo y fortalecidos por la regionalidad, algo que podría interpretarse como la difusión de la europeidad.

Como ya se ha mencionado, el proceso de integración regional en Europa alcanza también al sector de la Educación Superior, teniendo el Proceso de Bolonia la plena movilidad de los actores académicos y la consolidación de una Europa del conocimiento, ${ }^{16}$ como parte de un complejo movimiento de europeización de la Educación Superior con miras a formar un Espacio Europeo de Educación Superior (EEES) y la armonización de los sistemas nacionales de educación en Europa.

\section{ENLACES: POTENCIAL CATALIZADOR DE LA REGIONALIDAD Y DE LA INTEGRACIÓN EN AmÉrica LATINA y EL CARIBE}

El lanzamiento del ENLACES fue una de las más importantes resoluciones de la CRES 2008, ${ }^{17}$ que tuvo lugar en Cartagena de Indias (Colombia). Esta resolución colectiva en favor del lanzamiento del ENLACES está consignada en la Declaración oficial de la CRES 2008 que, según José Renato de Carvalho, sucesor de Ana Lúcia Gazzola en la dirección del IESALC-UNESCO, 
(...) concluye planteando la integración y la cooperación académica regional como estrategias básicas para la construcción de una Educación Superior de calidad, inclusiva y pertinente en los países de América Latina y el Caribe (...). Con el propósito de incentivar ese proceso, la CRES propuso la creación del Espacio de Encuentro Latinoamericano y Caribeño de Educación Superior (ENLACES), definió sus objetivos y estableció las estrategias de trabajo a ser implementadas (2009, p. 1).

Carvalho recuerda que, históricamente, existe el registro de otras iniciativas de integración regional como la propuesta por los dirigentes del MERCOSUR, en 1992, y por Ministros de Educación en Europa por intermedio de la Declaración de Bolonia de 1999. Sin embargo, Carvalho subraya que:

Varios factores diferencian la experiencia europea y la propuesta del ENLACES: el contexto histórico e institucional del
proceso de integración europeo, que asegura una fuerte estructura institucional y financiera, la existencia de un sistema
universitario consolidado y con alto desempeño académico en varios países y el compromiso de los Estados nacionales
con las propuestas planteadas en la Declaración de Bolonia. Obviamente, América Latina y el Caribe presentan un
contexto institucional distinto al europeo. Los procesos de integración regional aún son tímidos y reducidos, no existe
una organización supranacional capaz de movilizar y asegurar la adhesión de países a programas de carácter regional, faltan
recursos y prevalecen además, enormes deficiencias entre los sistemas de Educación Superior en la región, caracterizado por
una extrema diversidad de las estructuras institucionales de los sistemas nacionales de Educación Superior en América Latina
y el Caribe $(2009$, p. 1).

No es ocioso resaltar que la regionalización de los campos de la Educación Superior en Europa, a través del Proceso de Bolonia, cubre un territorio más amplio (48 países) que la Unión Europea (28 países), viene siendo estimulada por las autoridades regionales, como por ejemplo las políticas de movilidad estudiantil de la Comisión Europea, como en el caso del Programa ERASMUS (sigla en inglés European Region Action Scheme for the Mobility of University Students) o el Plan de Acción de la Comunidad Europea para la movilidad de los estudiantes universitarios, que de 1987 a 2013 promovió la movilidad de más de 3 millones de estudiantes de 4.000 instituciones de Educación Superior, con sede en 37 países. Este programa pasó a ser denominado como Proyecto Erasmus + por la Comisión Europea y definió ó para el período 2014 a 2020 un presupuesto de 14.700 millones de euros para la movilidad de más de 4 millones de personas, de las cuaes 2 millones son estudiantes (2 millones) y el resto profesores, funcionarios y jóvenes aprendices (EUROPEAN COMISION, 2017). En este período el programa pasa a cubrir, actividades de educación, formación y juventud, tanto a escala regional europea (internamente) como para la cooperación internacional extraeuropea. Erasmus + incluye algunos de los programas realizados por la Comisión Europea durante el período 2007-2013: Programa de Aprendizaje permanente, Programa Juventud en Acción, Programa Erasmus Mundus, Tempus, Alfa, Edulink y otros programas de cooperación en Educación Superior de países extraeuropeos.

En el ámbito del MERCOSUR Educacional, otra política regional de movilidad académica, es el Programa MARCA (Programa Movilidad Académica Regional para los Cursos Acreditados por el Mecanismo de Acreditación de Cursos Superiores en el Mercosur) que cubre una región mayor que los territorios de los Estados miembros del Mercosur (Argentina, Brasil, Paraguay, Uruguay y Venezuela), involucrando a diez países: Argentina, Bolivia, Brasil, Colombia, Chile, Ecuador, Paraguay, Perú, Uruguay, Venezuela). Esta política regional promovió desde 2006, de acuerdo con el portal del Programa MARCA, la movilidad de cerca de 2.000 estudiantes provenientes de 141 IES de la totalidad de los países participantes del Programa Marca (MARCA, 04 de septiembre de 2018).

El ENLACES, que representaría una política de movilidad estudiantil para los países de América Latina y el Caribe, es todavía una promesa. En efecto, los ejes de esta política regional no se han modificado. Se trata más de una plataforma virtual que estimularía esta política, que de un compromiso firme de movilidad. Según lo declarado en el portal del Programa con sede en IESA, el Espacio de Encuentro Latinoamericano y Caribeño de Educación Superior (ENLACES), es una plataforma regional de responsabilidad de todos sus participantes. ${ }^{18}$ Sin embargo, desde su creación está siendo "gestionado y administrado por UNESCOIESALC, bajo la supervisión de la Comisión de Seguimiento que buscará articular e involucrar a los actores interesados en los proyectos que la estructuran y conforman "(ENLACES, 03 de septiembre de 2018, p. 1). 
Esta comisión de seguimiento del ENLACES está formada por ocho representantes de entidades y asociaciones latinoamericanas y caribeñas y tiene las funciones de "consulta, apoyo técnico, supervisión y de hacer operativos el desarrollo de la agenda de trabajo para la construcción y desarrollo del ENLACES "(ENLACES, 03 de septiembre de 2018; p. 1$)^{19}$

Para la aplicación de esta agenda de solidaridad académica en América Latina y el Caribe, que podría denominarse de colaboración Sur-Sur, nuestra sugerencia sería formar un Fondo Regional para la financiación de la movilidad regional. Es que, incluso la internacionalización solidaria necesita ser financiada y este fondo para la movilidad estudiantil requiere del compromiso de los Estados participantes para ser abastecido. Sin financiación pública no es posible la promoción del bien público. Como ha dicho Albert Hirshman (1973), autor del clásico Salida, Voz y Lealtad, sin financiamiento público es muy posible que el mal público gane y, además, que los actores sociales ligados al sector privado mercantil avancen en el dominio sobre los campos de la Educación Superior en América Latina y el Caribe, especialmente bajo el mando de los fondos privados con sede en los países centrales, principales promotores de la transnacionalización y la mercantilización de la Educación Superior (Azevedo, 2015). ${ }^{20}$

A propósito, si los actores sociales identificados con el bien público en América Latina y el Caribe no toman las debidas providencias para el cumplimiento de lo acordado en las CRES 2008 y CRES 2018 y para poner en marcha el ENLACES, este programa puede incluso ser recuperado por proveedores privados transnacionales de educación superior y servir como plataforma para la promoción del capitalismo académico ${ }^{21}$ (Slaughter y Leslie, 1997; Azevedo, 2015). Es decir, a partir del proyecto de movilidad académica y de internacionalización de la Educación Superior en América Latina y el Caribe, el ENLACES puede convertirse en una plataforma de soporte de la transnacionalización de la Educación Superior.

En el mismo sentido de preocupación, también es importante velar por que ENLACES no se convierta en un apéndice o una sucursal del Programa Europeo Erasmus +, como se ha ensayado con el Programa de movilidad Mercosur, que realizó su convocatoria en 2010 con apoyo financiero de la Comisión Europea (Azevedo, 2014a). ${ }^{22}$ Cabe recordar que uno de los objetivos del Proceso de Bolonia es el de promover la capacidad de atracción de estudiantes extranjeros, algo consistente con la estrategia de Lisboa y la Estrategia Europa 2020. Según advierte Marco Antonio Días:

La Unión Europea no oculta que el financiamiento generoso de programas como Erasmus Mundus tiene como uno de sus principales objetivos el reclutar los mejores cerebros de otras partes del universo. Para alcanzar esto, estimula algo que en principio sería positivo, es decir, la creación de doctorados conjuntos, acompañados por ambiciosos programas de becas. Pero en la medida en que el objetivo de Erasmus Mundus — según confirma Sarah Piovezan en un artículo publicado en Le Monde, el 29 de enero de 2008 - es el de reclutar explícitamente los mejores cerebros, al final, más «cooperación» puede significar más estímulo al éxodo de cerebros (2016, p. 16).

Para hacer frente a un escenario privatista y de fuga de cerebros, lo que no sería novedoso en la región, los actores sociales que defienden la Educación Superior como un bien público y están comprometidos con la solidaridad, la interculturalidad, el respeto mutuo y la justicia social en América Latina y el Caribe, necesitan urgentemente r operacionalizar la Comisión de Seguimiento de ENLACES (y/o un grupo de cumplimiento) para la aplicación y ejecución de lo acordado en las CRES 2008 y 2018, tal como mencionamos al inicio de este artículo. De esta manera gobiernos nacionales, instituciones de Educación Superior, redes, estudiantes, investigadores, movimientos sociales, autoridades, organizaciones e instituciones regionales y otros actores sociales interesados en la defensa de la integración solidaria en América Latina, podrán elegir el ENLACES como prioridad política. Este sería un "desenlace" necesario para el ENLACES (con las disculpas por el pobre juego de palabras) - para catalizar el desarrollo de una sustantiva regionalidad latinoamericana y caribeña, fundamento intercultural para una orgánica regionalización en el Sur Global.

Considerando que, aunque lentamente, está en curso la formación de un campo social regional de Educación Superior y, siendo esta regionalización un proceso de convergencia, no puede dejar de considerarse 
que a pesar de tratarse de un campo social regional en integración, involucra tensiones y luchas entre los actores sociales, pero también presupone la consecución de consensos y de una sustantiva regionalidad. Un cuerpo de cultura que constituye potencialmente una amalgama de solidaridad para la identidad latinoamericana y caribeña en construcción.

\section{CONSIDERACIONES FINALES}

La Educación Superior ha sido considerada como parte fundamental en cualquier proceso de integración regional. Una región integrada tiene influencia en las relaciones socio-políticas y espaciales nacionales, supranacionales (regiones europeas), extrarregionales (otras regiones) y globales (Robertson, et. al, 2016). La formación de un Espacio Europeo de Educación Superior (EEES), afecta a otros países y regiones y puede interpretarse, sobre la base de la teoría de los campos de Bourdieu (2011), como una gradual (con)fusión controlada de campos sociales académicos junto con la preservación orgánica de los campos nacionales de Educación Superior. Más allá de esto, la armonización y la convergencia para la regionalización presuponen un juego de comparabilidad y una continua evaluación. ${ }^{23}$

Como hemos visto, el ENLACES en América Latina y el Caribe juega potencialmente un papel fundamental para la formación de una cultura regional o, como recuerda Hettne (2005), de una regionalidad. Los campos de Educación Superior de cada país, en proceso de formación de un campo social regional académico, pueden contribuir con la formación de una sustantiva regionalidad. Para ello, son necesarias políticas y programas (por iniciativa nacional y también regional) de cooperación y movilidad académicas (de estudiantes, docentes y técnicos), teniendo por principios: la solidaridad, el respeto mutuo, la reciprocidad y la interculturalidad.

Finalmente, al tratarse de un proceso de convergencia de políticas y de compatibilización de reglas de diversos campos sociales, la regionalización se internaliza a través de la convalidación nacional de las normas, reglas y regulaciones comunes impulsadas por los legisladores de cada Estado miembro. Según la correlación de fuerzas en los campos sociales, esta dinámica, cataliza la institucionalización política y cultural comunitaria, la regionalidad y el sentimiento de pertenencia regional. Pero, contradictoriamente, aun siendo la regionalización, un proceso de convergencia, consiste en un juego de fuerzas no siempre explícito y formalizado. La formación de campos sociales “supra nacionales” consiste en un proceso tenso, en la medida en que todo campo social es un campo de fuerzas, sobre todo en escala regional. ${ }^{24}$

\section{REFERENCIAS}

Azevedo, M. L. N. (2014a.). The Bologna Process and higher education in Mercosur: ¿regionalization or Europeanization? International Journal of Lifelong Education, 33, (2), 411-427.

Azevedo, M. L. N. (2014b) A Integração Regional, a Estratégia Europeia de Desenvolvimento e algumas comparações com o Mercosul. Revista Latinoamericana de Educacación Comparada, RELEC, 5, $29-40$.

Azevedo, M. L. N. (2015). Transnacionalização e mercadorização da educação superior: examinando alguns efeitos colaterais do capitalismo acadêmico (sem riscos) no Brasil - A expansão privado-mercantil. RIESup-Revista Internacional De Educação Superior. (1), 86 - 102.

Azevedo, M. L. N. (2018). Regionalismo, regionalização e regionalidade: da integração pelas paz à Estratégia Europa 2020. En Barreyro, G.B.; Hizume, G.C. Regionalismos e inter-regionalismos na educação superior: projetos, propostas e influências entre a América Latina e a Europa. Cascavel: EdUnioete.

Best, E.; Christiansen, T. (2008). Regionalism in international affairs. In Baylis, J.; Smith, S.; Owens, P. (Eds.). The globalization of world politics. An introduction to international relations. (4th ed., pp. 434-449.). Oxford: Oxford University Press. 
Börzel, T. A. (2016). Theorizing regionalism: cooperation, integration, and governance. The Oxford Handbook of Comparative Regionalism. Political Science, International Relations, Comparative Politics online Publication. Oxford: Oxford University Press.

Bourdieu, P. (2011). O campo político. Revista Brasileira de Ciência Politica, 5, 193-216.

Carvalho, J.R. (2009). Enlaces: Una estrategia de cooperación e integración. Boletín IESALC Informa Edición Especial no. 200. Retrieved from www.iesalc.unesco.org.ve/dmdocuments/boletin_200_espanol.pdf

Comisión Europea. (2002). CECA - EKSF - EGKS - EKAX - ECSC - EHTY - EKSG 1952-2002. Luxembourg: Office des Publications Pfficielles des Communautés Européennes.

Comisión Europea. (2010) Europa 2020: Estratégia para um crescimento inteligente, sustentável e inclusivo. Bruxelas: Comissão Europe.

Comisión Europea. (2016) Erasmus +. Guia do Programa. Bruxelas: Comissão Europeia.

Consejo Europeo de Lisboa. (23 y 24, marzo, 2000). Conclusões da Presidência. Lisboa, Disponível em: www.consilium.europa.eu/ueDocs/cms_Data/docs/pressData/pt/ec/00100-r1.p0.htm

Dale, R.; Robertson, S. L. (2009). Globalisation \& Europeanisation in Education. Oxford: Symposium Books.

Dale, R. (2004). Globalização e educação: demonstrando e existência de uma cultura educacional mundial comum" ou localizando uma "agenda globalmente estruturada para a educação? Educação e Sociedade, 25, (87), 423-460.

Dale, R. (2009). Contexts, constraints and resources in the development of European education space and European education policy. In: Dale, R. \& Robertson, S.L (Eds.). Globalisation \& Europeanisation in Education. Oxford: Symposium Books.

Declaración CRES 2008. (s.f.). Recuperado de www.iesalc.unesco.org.ve

Declaración. CRES 2018. (s. f.). Recuperado de www.iesalc.unesco.org.ve

Dias, M.A. (septiembre, 2016). Enseñanza superior como bien público:perspectivaspara el centenário de la Declaración de Córdoba. Ponencia presentada en Seminario en conmemoración de los 25 años de la Asociación de Universidades Grupo Montevideo (AUGM), Montevideo, Uruguay. Recuperado de http://grupomontevideo.org/sitio/wp-c ontent/uploads/2017/08/Marco-Antonio-Rodrigues-Dias_ES-como-bien-p\%C3\%BAblico.pdf

ENLACES (s.f.) Sobre Enlaces. Recuperado de https://www.iesalc.unesco.org.ve/index.php?option=com_content\& view $=$ category $\&$ id $=133 \&$ Itemid $=631 \&$ lang $=e s$

ENLACES (2018) INFORME DEL COMITE EJECUTIVO PROVISIONAL DEL ESPACIO LATINOAMERICANO Y CARIBEÑO DE EDUCACION SUPERIOR A LA PRIMERA CONFERENCIA GENERAL ORDINARIA DE ENLACES http://espacioenlaces.org/wp-content/uploads/2018/07/ENLAC ES-informe-trayectoria.pdf

Esping-Andersen, G. (1991) As três economias políticas do welfare state. Lua Nova, 24,. Disponível em https://www .scielo.br/scielo.php?script=sci_arttext\&pid=S0102-64451991000200006\&lng=en\&nrm=iso

Eurogoogle (s.f.). Spill-over. Disponível em: https://euroogle.com/dicionario.asp?definition=1347

European Comission. (2017). ERASMUS+ The EU programme for education, training, youth and sport (2014-2020). Bruxelas: Comissão Europeia.

Fernandes, N. (maio, 2010). Crise na Europa reflete socialização das perdas de 2008, diz Dany Rappaport. O Estado de S. Paulo, Disponível em http://economia.estadao.com.br/noticias/economia,crise-na-europa-reflete-socializacaodas-perdas-de-2008-diz-dany-rappaport, 17588,0.htm

Haas, E. B. (1958). The uniting of Europe. Political, Social, and Economic Forces 1950-1957. Stanford: Stanford University Press.

Hettne, B. (2005). Beyond the 'new' regionalism. New Political Economy, 10, (4), 543-571.

Hirschman, A. (1973). Saída, voz e lealdade: reações ao declínio de firmas, organizaçôes e estados. São Paulo: Perspectiva.

IESALC-UNESCO (2018). Reunión Nacional Técnica Especializada para avanzar con el Plan de Acción de la III CRES. Portal do IESALC. Recuperado de www.iesalc.unesco.org.ve/index.php?option $=$ com_content\&view $=$ article\&id $=4191$ :reunion - nacional- 
tecnica-especializada-para-trabajar-el-plan-de-accion-de-la-cres-2018\&catid=100:enportada\&Itemid $=449 \&$ lang $=\mathrm{es}$

IESALC (s.f.). CRES 2008. Recuperado de www.unesco.org.ve/index.php? option $=$ com_content\&view $=$ article\&id $=365 \&$ Itemid $=423 \&$ lang $=$ pt

Kauppi, N. (2003). Bourdieu's political sociology and the politics of European integration. Theory and Society, 32, (5-6), 775-789.

Laursen, F. (2008). Theory and practice of regional integration. The Jean Monnet Chair Miami-Florida European Union Center of Excellence.. Published with the support of the EU Commission.Jean Monnet/Robert Schuman Paper Series, 8, (3).

MARCA (s.f.). Convocatórias. Programa Mobilidade Acadêmica Regional para os Cursos Acreditados pelo Mecanismo de Acreditação de Cursos Superiores no Mercosul . Recuperado de http://programamarca.siu.edu.ar/ programa_marca/convocatoria_programa_marca.html

Melo, S. (2016). Different regionalisms, one European higher education regionalization: the case of the Bologna Process. In Robertson, S. L. et al. Global regionalisms and higher education: projects, processes, politics. (pp. 49-64) Cheltenham, UK; Northampton, MA, USA: Elgar.

Offe, C. (2003). The European model of "social" capitalism: can it survive European integration? The Journal of Political Philosophy, 11, (4), 437-469.

Polanyi, K. (1980). A grande transformação: as origens de nossa época. (3. ed.). Rio de Janeiro: Campus.

Robertson, S. L. et al. (2016). Global regionalisms and higher education: projects, processes, politics. Cheltenham, UK; Northampton, MA, USA: Elgar.

Robertson, S.L.; Dale, R. (2017). Comparando Políticas em um Mundo em Globalização: reflexões metodológicas Educação \& Realidade, Porto Alegre, 42, (3), 859-876, jul./set. 2017. Recuperado de dx.doi.org/10.1590/2175-623670056

Robertson, S. L.; Azevedo, M. L. N.; Dale, R. (2016). Higher education, the EU, and the cultural political economy of regionalism. In Robertson, S. L. et al. (Eds.). Global regionalisms and higher education: projects, processes, politics (pp. 24-48). Cheltenham, UK; Northampton, MA, USA: Elgar, 2016.

Schumpeter, J. A. (1961). Capitalismo, socialismo e democracia. Rio de Janeiro: Fundo de Cultura.

Schumpeter, J. A. (1997). Teoria do Desenvolvimento Econômico: uma investigação sobre lucros, capital, crédito, juro e o ciclo econômico. São Paulo: Nova Cultural.

Slaughter, S.; Leslie L. L. (1997). Academic Capitalism: Politics, Policies, and the Entrepreneurial University. Baltimore: Johns Hopkins University Press.

Söderbaum, F. (2008).The waning tate, conceptualizing the Region and Europe as a Global Actor. Theory Talks \#19. Disponível e www.theorytalks.org/2008/10/theory-talk-19.html

Söderbaum, F. (2015). Early, old, new and comparative regionalism: The scholarly development of the field. KFG Working Paper Series, Kolleg-Forschergruppe (KFG) "The Transformative Power of Europe", Berlin; Freie Universität.

Unión Europea. (s.f.) a. Instituições e outros organismos da UE. Disponível em: http://europa.eu/about-eu/institutio ns-bodies/index_pt.htm

Unión Europea (s.f.) b. Como funciona a UE. [s.d.]b. Disponível em: http://europa.eu/about-eu/index_pt.htm

Unión Europea (s.f.) c. Tratado que institui a Comunidade Europeia do Carvão e do Aço: Tratado CECA. Disponível em: http://europa.eu/legislation_summaries/institutional_affairs/treaties/treaties_ecsc_pt.htm

Valle, D.; Perrota, D. (2018). Las redes académicas: Entre los discursos y las políticas para la internacionalización con integración regional. En Korsunsky, L. et al. Internacionalización y producción de conocimiento: el aporte de las redes académicas. ( $1^{\mathrm{a}} \mathrm{ed}$.). Ciudad Autónoma de Buenos Aires: IEC - CONADU-CLACSO. 


\section{Notas}

1 Con todo, debe reconocerse que el ENLACES puede ser incluido en el Plan de Acción de la CRES 2018 que aún no está publicado. Según el portal del IESALC, el 29 de agosto de 2018, se celebró una reunión técnica sobre el Plan de Acción. El 29 de agosto de 2018 tuvo lugar en la sede de UNESCO-IESALC, en Caracas, Venezuela, una Reunión Nacional Técnica Especializada del Plan de Acción de la III Conferencia Regional de Educación Superior (CRES 2018). Asistieron a esta reunión rectores y representantes de universidades venezolanas con la finalidad de discutir y analizar el documento preliminar, relativo a contenidos propositivos y su exposición operativa en la región para el período 2018-2028 (IESCALC, 29 agosto 2018).

2 La Unión Europea- UE "tiene un marco institucional único: - las grandes prioridades de la UE se definen por el Consejo Europeo, que reúne a dirigentes nacionales y de la UE; los eurodiputados representan a los ciudadanos europeos en el Parlamento Europeo y son elegidos directamente por ellos; los intereses de la UE en su conjunto son defendidos por la Comisión Europea, cuyos miembros son designados por los gobiernos nacionales; los países defienden sus propios intereses nacionales en el Consejo de la Unión Europea " (Unión Europea, [s.f.] a). Entre otras instituciones, el Banco Central Europeo (BCE), el Tribunal de Cuentas Europeo y el Tribunal de Justicia, que "interpreta el Derecho de la UE para garantizar su aplicación uniforme en todos los Estados miembros. Además, resuelve los litigios entre los gobiernos nacionales y las instituciones europeas. Particulares, empresas y organizaciones pueden recurrir al Tribunal si consideran que sus derechos han sido infringidos por una institución europea "(Unión Europea [s.f.] a).

3 En parte, argumentos aquí presentes figuran en el capítulo "Regionalismo, regionalización y regionalidad: de la integración por la paz a la Estrategia Europa 2020" (Azevedo, 2018).

4 Ésta y las demás versiones procedentes de las lenguas inglesa y francesa son del autor del artículo.

5 El capital cultural específico de la región, potencialmente limitado y regulado al quantum poseído, es convertible en otras especies de capital (político, económico, social, etc.).

6 La posición de los actores sociales en los campos es determinada por la relación de fuerzas en el espacio social y se define por la posesión de capital específico y por el habitus. Kauppi observa que "los recursos políticos y económicos disponibles para los grupos que ocupan posiciones centrales en la Euro-esfera (para usar la expresión acuñada por Dusan Sidjanski, los habilita cada vez más para determinar las reglas del juego político transnacional por intermedio, por ejemplo, de configuraciones institucionales (para ilustrar: reforzando la posición de la Comisión Europea) y la imposición de nuevos principios de clasificación social [...] (el 70\% de la nueva legislación procede del nivel europeo). Esta concentración de recursos también transforma las culturas políticas de los subcampos políticos nacionales y regionales, como demuestra el caso del Parlamento Europeo" (Kauppi, 2003, p.783).

7 De acuerdo con Hass (1958, p. 16) "la integración política es el proceso por el cual los actores políticos, en varios contextos nacionales distintos, son persuadidos a cambiar sus lealtades, expectativas y actividades políticas hacia un nuevo centro cuyas instituciones poseen o exigen jurisdicción sobre los estados nacionales preexistentes".

8 El portal Euroogle, que reúne diccionarios europeos, define de la siguiente manera la expresión spill-over: "El efecto spillover es entendido como los efectos positivos o negativos que una determinada actividad puede generar sobre otros que no se encuentran directamente involucrados en ella. El ruido de una lavadora genera un efecto spill-over negativo sobre los vecinos. La limpieza de la calle genera un efecto spill-over positivo sobre los residentes. En lo que se refiere a la política de cohesión, los Fondos estructurales se asignan a las 271 regiones europeas, siendo superiores a las regiones menos desarrolladas en detrimento de las regiones en transición y de las regiones más desarrolladas. Sin embargo, una parte importante de los fondos asignados a algunas regiones pueden transferirse a las regiones más desarrolladas con el efecto spill-over, especialmente en los casos en que un proyecto de inversión realizado en este tipo de regiones tiene efectos positivos en otras regiones del Estado Miembro. A pesar de que las regiones más desarrolladas ya tienen derecho a una dotación financiera, podrían beneficiarse de fondos adicionales transferidos de otras regiones a inversiones que tendrán un efecto difusor a escala nacional y dotar al país de mayor competitividad. Cada Estado miembro será responsable de definir, en sus programas operativos, los importes que podrán utilizarse con arreglo al efecto spill-over (Eurogoogle, [s.d]].

9 De acuerdo con datos del portal oficial de la Unión Europea (UE), 28 países constituyen esta especial asociación económica, política y social. Históricamente, "la UE comenzó a raíz de la Segunda Guerra Mundial, con el fin de fomentar la cooperación económica en Europa, partiendo del supuesto de que los países con relaciones comerciales se volvían económicamente dependientes, reduciendo así los riesgos de conflicto. De esta cooperación económica resultó la creación de la Comunidad Económica Europea (CEE) en 1958, inicialmente constituida por seis países: Alemania, Bélgica, Francia, Italia, Luxemburgo y los Países Bajos [...]. Lo que empezó por ser una unión meramente económica, ha evolucionado hacia una organización que abarca ámbitos de intervención variados que van desde la ayuda al desarrollo a la política ambiental hacia......... El cambio de nombre de CEE para la Unión Europea (UE) en 1993 refleja esta evolución "(Unión Europea [s.f] b). 
10 Es importante señalar que, antes de la creación del bloque europeo, el Benelux, acrónimo formado por las iniciales de tres países miembros -Bélgica, Holanda (Netherlands) y Luxemburgo-, fue un acuerdo firmado en 1943 durante la Segunda Guerra Mundial, con el objetivo de promover la integración económica y el comercio entre los miembros.

11 De acuerdo con el Portal de la Unión Europea, "la primera organización comunitaria surgió poco después de la Segunda Guerra Mundial, cuando se consideró necesario reconstruir económicamente el continente europeo y asegurar una paz duradera. Así nació la idea de reunir la producción franco-alemana de carbón y de acero y que surgió la Comunidad Europea del Carbón y del Acero (CECA). Esta opción obedeció a una lógica no sólo económica como política, ya que estas dos materias primas constituían la base de la industria y el poder de estos dos países. El objetivo político subyacente era claramente el refuerzo de la solidaridad franco-alemana, el alejamiento del espectro de la guerra y la apertura de una vía hacia la integración europea "(Unión Europea, [s.f] c).

12 Claus Offe (2003), en un pertinente artículo titulado The European model of 'social' capitalism: can it survive European integration? señala que la Europa del Bienestar podría estar en riesgo, y advierte que en la integración europea puede estar en peligro la europeidad social (la regionalidad social) en función de la europeidad de la integración capitalista (la regionalidad del mercado).

13 "La estrategia de Lisboa ha confirmado el punto de vista neoliberal que la educación superior contribuye al bienestar socioeconómico de la región, la construcción y la garantía de capital humano para una economía basada en el conocimiento" (Robertson; Azevedo; Dale, 2016, p 30) [...]. "Esta nueva concepción combinó un paradigma schumpeteriano, centrado en la innovación y el espíritu emprendedor, en la teoría del capital humano, en la economía del lado de la oferta y en condiciones de comercio más libres - todas las condiciones consideradas importantes para el mercado mundial que avanza a través de las fronteras regionales" (Robertson; Azevedo; Dale, 2016, p. 33).

14 El prefacio de la Estrategia Europa 2020, firmado por José Manuel Barroso, anuncia que la "prioridad inmediata es asegurar una salida exitosa de la crisis" (Comisión Europea, 2010, p.2). Barroso afirma que el objetivo de la Estrategia Europa 2020 es "crear más empleo y asegurar mejores condiciones de vida. Esta estrategia demuestra la capacidad de Europa para generar un crecimiento inteligente, sostenible e inclusivo "(Comisión Europea, 2010, p.2). La Estrategia Europa 2020, como se ha dicho anteriormente, parece inspirarse en la teoría del desarrollo de Schumpeter y en la teoría del capital humano. Los formuladores de esta última, fundados en el individualismo metodológico, defienden la idea de que las asignaciones para la calificación, la educación (y el emprendimiento) causan reflejos directos en el aumento salarial y en el crecimiento económico, significando, a nivel individual, movilidad social, aumento de la productividad y mayores ganancias salariales y, en un plano macroeconómico, desarrollo regional - el corazón de la Estrategia Europa 2020.

15 De acuerdo con Robertson et al, "trabajar con los nuevos regionalismos ha sido importante no sólo porque hizo avanzar el trabajo conceptual, considerando las regiones como formadas tanto hacia adentro y afuera. También porque los nuevos regionalismos colocaron a las regiones como bloques, en el centro del orden mundial "(Robertson et al., 2016, p.10).

16 De acuerdo con Azevedo, "el proceso de Bolonia, aunque no es una iniciativa formal de la Unión Europea (UE), fortalece la regionalización y la integración de la educación en Europa, sobre todo a través de la contenida en el" Tratado de Lisboa". El EEES amplía la idea de "europeidad" y amplía la territorialidad europea a un ámbito más amplio que la propia UE. La adhesión formal de 47 países [en 2016, 50 países] al Proceso de Bolonia supera las fronteras de los 27 países de la UE [en 2016, 28 países]. [...] Otras regiones y países no europeos han intentado establecer acuerdos de cooperación y emprender reformas que convergen con el modelo proclamado por el Proceso de Bolonia. Así, la europeización de la Enseñanza Superior es un fenómeno que ocurre en y más allá de Europa "(2014a, p.2). Además, para Melo, "el Proceso de Bolonia es un caso de regionalismo informal, en el que la capacidad de acción política como unidad regional ha sido desarrollada por una constelación de actores cuya experiencia es la política de Educación Superior" (2016, p. 61).

17 "CRES 2008 fue patrocinada por UNESCO-IESALC, Ministerio de Educación de Brasil, Ministerio de Educación Nacional de Colombia, Ministerio de Educación, Política Social y Deporte de España, Secretaría de Educación Pública de México, Ministerio del Poder Popular para la Educación del Gobierno Bolivariano de Venezuela. (CEPAL) de Colombia. Recibió apoyo de la Asociación Colombiana de Universidades (ASCUN) "(IESALC-CRES 2008, 04 de septiembre de 2018).

18 El portal de ENLACES responde a la pregunta sobre ¿quiénes participan? enumerando los siguientes participantes: Ministerios de Educación; Ministerios de Educación Superior; Instituciones Gubernamentales; Instituciones de Educación Superior (IES); Redes y Asociaciones de Instituciones de Educación Superior; Agencias Nacionales e Internacionales de Desarrollo de la Educación Superior y de Ciencia, Tecnología e Innovación; Individuos: docentes, investigadores, estudiantes, trabajadores técnico-administrativos, etc. (ENLACES, 03 de septiembre de 2018).

19 Miembros de la III Comisión de Seguimiento de ENLACES (2012): Instituto Internacional de la UNESCO para la Educación Superior en América Latina y el Caribe (UNESCO-IESALC) - Pedro Henriquez Guajardo; Asociación Nacional de los Dirigentes de las Instituciones Federales de Enseñanza Superior (ANDIFES) - Joao Luiz Martins; Asamblea Nacional de Rectores - Orlando Velasquez Benites; Organización Continental Latinoamericana y Caribeña de Estudiantes (OCLAE) - Reynier Limonta; Asociación de Universidades Grupo Montevideo (AUGM) - Targino de Araújo Filho; Consejo Superior Universitario Centroamericano (CSUCA) - Gustavo García de Paredes; Unión de 
Universidades de América Latina y el Caribe (UDUAL) - Roberto Inván Escalante Semerena; Asociación Nacional de Universidades e Instituciones de Educación Superior (ANUIES) - Rafael López Castañares.

20 "(...) la palabra capitalismo connota la propiedad privada de los factores de producción-tierra, trabajo y capital- y considerar funcionarios de universidades públicas como capitalistas, a primera vista, parece una flagrante contradicción. Sin embargo, el capitalismo también se define como un sistema económico en el que las decisiones de asignación se mueven por las fuerzas del mercado "(Slaughter, S.; Leslie 1997, p.9).

21 En tiempos de Economía de Plataforma, no sería exagerado inferir que el ENLACES, en caso de que ocurra una supuesta supremacía de actores privados de los campos de la Educación Superior, puede convertirse en un espacio de promoción del "capitalismo académico de plataforma". En el caso de capitalismo académico de plataforma, fueron presentadas por Aliandra Lazzari Barlete y Mário Azevedo en una comunicación titulada Higher education, platform and the academic profession in Latin America: a case for platform academic capitalism, en el evento "Capitalism, Social Science and the Platform University", en la Universidad de Cambridge, el 13 de diciembre de 2018.

22 El Programa Movilidad Mercosur fue un ejemplo de influencia europea en la movilidad académica regional en el Mercosur. Azevedo (2014a) comprendió como una europeización de la Educación Superior en el MERCOSUR. La Guía del programa Erasmus + clasifica 10 regiones con países asociados que pueden participar en el programa europeo. La región 8 está compuesta por los siguientes países de América Latina y el Caribe: Argentina, Bolivia, Brasil, Chile, Colombia, Costa Rica, Cuba, Ecuador, El Salvador, Guatemala, Honduras, México, Nicaragua, Panamá, Paraguay, Perú, Uruguay, Venezuela (Comisión Europea, 2016, p. 25).

23 Esto porque, de acuerdo con Bourdieu, "la noción de campo político [...] permite construir de manera rigurosa esa realidad que es la política o el juego político. A continuación, permite comparar esta realidad construida con otras, como el campo religioso y el campo artístico. Como todos saben, en las ciencias sociales, la comparación es uno de los instrumentos más eficaces, al mismo tiempo de construcción y de análisis "(2011, p. 194).

24 Es importante notar que la regionalización ocurre en sociedades de clase en que, por un lado, se presentan actores identificados con los principios de la solidaridad, la cooperación y la justicia social, y de otro, aquellos que apoyan proyectos basados en la competencia, el individualismo y los mercados. 